\title{
Acute Stroke Imaging Part II: The Ischemic Penumbra
}

\author{
K. Butcher, D. Emery
}

\begin{abstract}
In acute ischemic stroke, the volume of threatened but potentially salvageable tissue, i.e. the ischemic penumbra, is critical to the success of all acute therapeutic interventions, most notably thrombolysis. Despite the availability of both CT and MRI based techniques to detect and assess the penumbra, advanced imaging of this type remains under-utilized. Although the optimal selection criteria are still being refined and technical improvements are ongoing, rapid imaging of the penumbra appears to be the most promising approach to expanding the acute thrombolysis population, as well as tailoring treatment based on specific pathophysiological findings. This second article in a two-part series reviews current evidence for penumbral-based treatment selection and discusses the barriers to implementation of these advanced imaging techniques in acute stroke management protocols.
\end{abstract}

RÉSUMÉ: Imagerie de l'accident vasculaire cérébral aigu, deuxième partie : la pénombre ischémique. En phase aiguë de l'accident vasculaire cérébral, le volume du tissu à risque mais potentiellement viable, c'est-à-dire la pénombre ischémique, est très important pour le succès de toute intervention thérapeutique dont la thrombolyse. Malgré la disponibilité de techniques basées sur la tomodensitométrie et sur l'imagerie par résonance magnétique pour détecter et évaluer la pénombre, ces types d'imageries demeurent sous-utilisées. Bien que les critères de sélection optimaux ne soient pas encore bien définis et que la technologie se perfectionne continuellement, l'approche la plus prometteuse pour augmenter la population de patients qui subissent une thrombolyse en phase aiguë ainsi que pour individualiser le traitement sur la base de constatations physiopathologiques spécifiques demeure l'imagerie rapide de la pénombre. Ce second article de cette série de deux articles revoit les données actuelles concernant le choix du traitement basé sur la pénombre et discute des barrières à l'utilisation de ces techniques avancées d'imagerie dans les protocoles de prise en charge de l'accident vasculaire cérébral aigu.

Can. J. Neurol. Sci. 2010; 37: 17-27

\section{Rationale for Penumbral Imaging}

The ischemic penumbra concept arguably represents the most successful translation of scientific laboratory results into the clinical stroke realm. The penumbra was originally defined by Symon et al in the late 1970 's, based on experimental stroke studies performed in baboons. ${ }^{1-3}$ These studies indicated a range of cerebral blood flow (CBF) values are present within the vascular territory of an occluded cerebral artery. Furthermore, various $\mathrm{CBF}$ values ranging from an upper threshold, where electrical silence is first observed, to a lower threshold associated with loss of cellular integrity were identified. ${ }^{3}$ Regions between these two flow thresholds were described as 'penumbral' (Figure 1). The ischemic penumbra has since been defined as brain tissue which is oligemic and functionally inactive, but structurally intact and therefore potentially salvageable. ${ }^{3}$ Subsequent nuclear medicine positron emission tomography (PET) studies indicated that there are $\mathrm{CBF}$ thresholds for penumbra (18-22 $\mathrm{ml} / 100$ $\mathrm{g} / \mathrm{min}$ ) and irreversibly injured infarct 'core' $(10 \mathrm{ml} / 100 \mathrm{~g} / \mathrm{min})$ in clinical stroke patients. ${ }^{4,5} \mathrm{~A}$ number of hemodynamic and metabolic changes occur following occlusion of a cerebral artery. Under ischemic conditions, vasodilation results in compensatory increases in cerebral blood volume $(\mathrm{CBV})$ in the penumbra, while $\mathrm{CBV}$ in the infarct core is lower than normal unaffected tissue. Changes in CBV as well as an increase in the oxygen extraction fraction may temporarily meet metabolic demands and delay infarction. ${ }^{6}$

The extent of the ischemic penumbra is critical to the success of any acute stroke therapy, including thrombolysis. Patients without a penumbra are unlikely to benefit from any acute tissue salvage therapies, as irreversible injury has already occurred. Conversely, patients with penumbral tissue may benefit from tissue salvage therapies, regardless of the duration of symptoms. Until recently identification of the ischemic penumbra in a

From the Division of Neurology (KB), Department of Diagnostic Imaging (DE), University of Alberta, Edmonton, Alberta, Canada.

Received June 22, 2009. Final Revisions Submitted August 17, 2009. Correspondence to: Ken Butcher, Division of Neurology, 2E3 WMC Health Sciences Centre, University of Alberta, 8440 112th St., Edmonton, Alberta, T6G 2B7, Canada. 


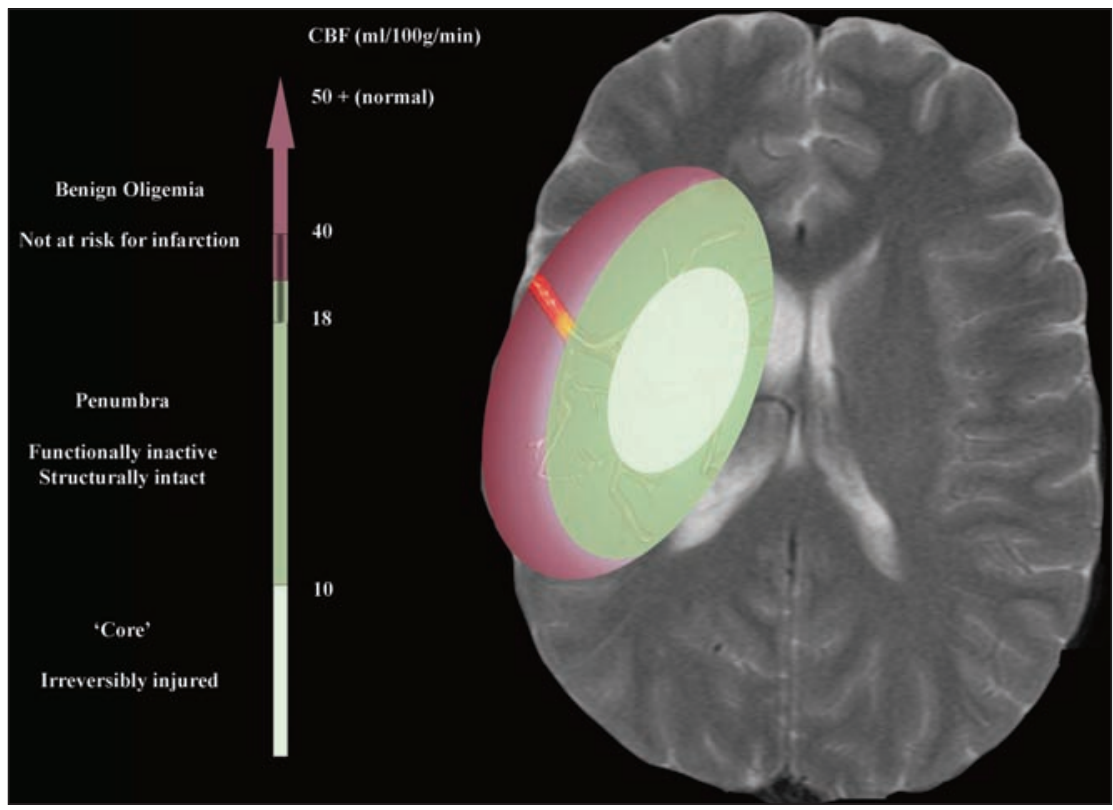

Figure 1: The ischemic penumbra. Following arterial occlusion (yellow denotes thrombus), cerebral blood flow (CBF) decreases non-uniformly throughout the territory of the affected vessel. Areas where CBF decreases below $10 \mathrm{ml} / 100 \mathrm{~g} / \mathrm{min}$ are irreversibly injured (infarct core). Tissue with CBF between 10 and approximately $20 \mathrm{ml} / 100 \mathrm{~g} / \mathrm{min}$ (absolute thresholds vary) is dysfunctional, but viable and potentially salvageable (ischemic penumbra). The penumbral is the target of all acute stroke therapies, including thrombolysis.

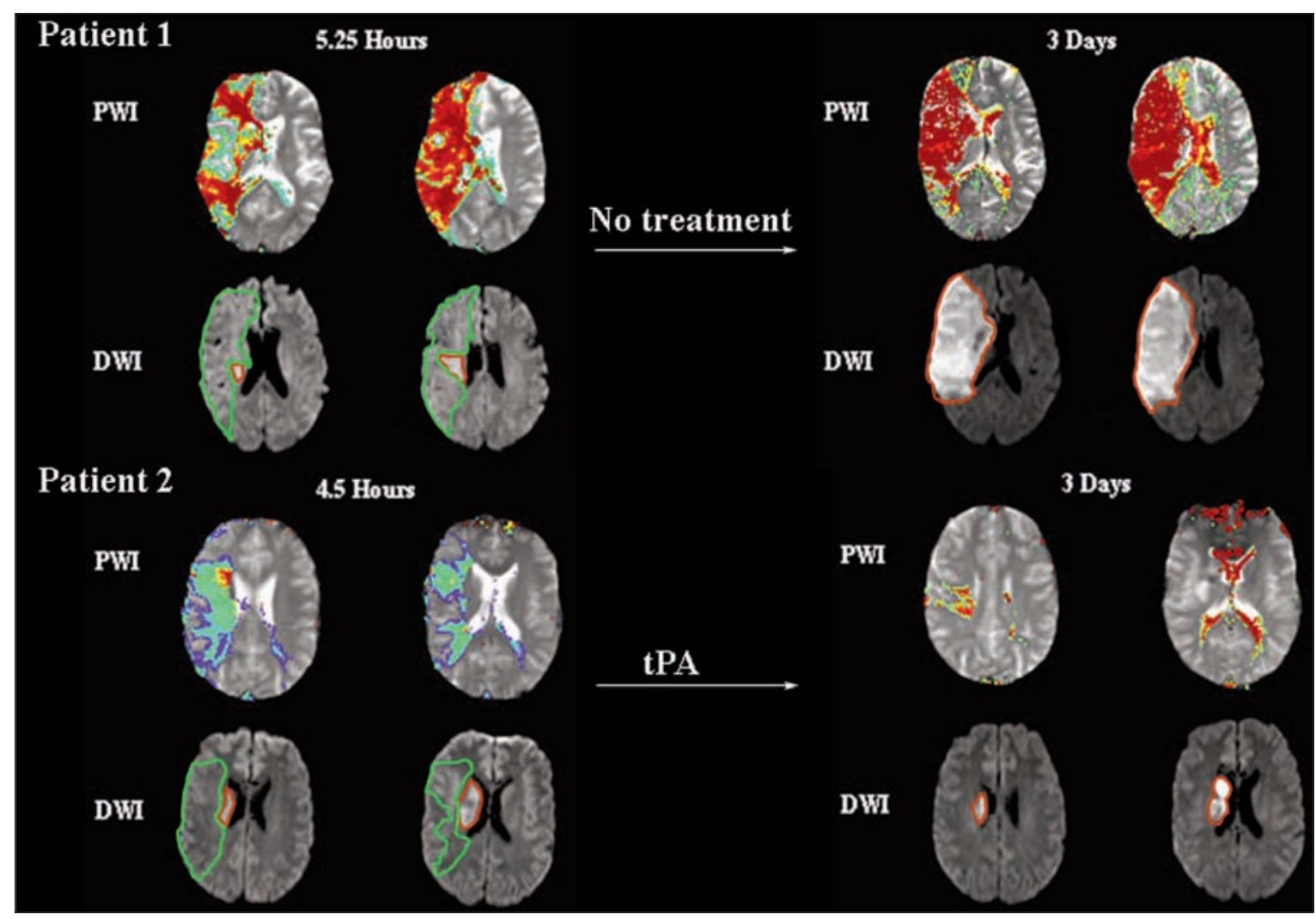

Figure 2: Examples of Perfusion-Diffusion weighted imaging (PWI-DWI) mismatch + pattern evolution. Both patients have large areas of decreased blood flow, demonstrated on PWI (green border). These are Tmax maps, which have been thresholded to include only areas of Tmax delay $>2 \mathrm{~s}$ $($ Tmax $+2 s)$, providing an objective measure of the hypoperfused volume. Both patients have smaller regions of infarction indicated on DWI (red border). In the absence of treatment (Patient 1; top), the infarct grows substantially. Thrombolysis (Patient 2; bottom) results in reperfusion and attenuation of infarct growth. 
timely fashion has been problematic, as the short half-life of tracer nucleotides renders nuclear medicine investigations (PET and SPECT) impractical in the acute setting. Modern imaging techniques, utilizing MRI or multi-modal CT, permit visualization of both irreversibly injured ischemic core regions and hypoperfused brain tissue that is structurally intact, but at risk for progression to infarction, i.e. the ischemic penumbra. ${ }^{7-9}$ Thus, the penumbra has become less of a theoretical construct and a more practical treatment target. ${ }^{10}$

\section{Imaging the Ischemic Penumbra: MRI}

As described in detail in Part I, CBF can be measured using Perfusion-Weighted Imaging (PWI) MRI. The extent of largely irreversibly injured tissue is easily assessed with DiffusionWeighted Imaging (DWI). Thus, an operational definition of the ischemic penumbra, based on the appearance of DWI and PWI in acute ischemic stroke patients has been developed (Figure 2). ${ }^{11}$ In this model, the area at risk is demonstrated by a PWI map, while the DWI lesion indicates the extent of largely irreversibly injured ischemic 'core' tissue. Mismatch between a larger PWI abnormality, indicated on one or more of the parametric maps, and a smaller DWI lesion has been postulated to represent the ischemic penumbra, in which tissue is at risk for infarction, but also potentially amenable to salvage (Table 1). ${ }^{12}$

The mismatch concept is certainly biologically plausible and there is a growing body of supportive evidence. Indeed, serial MRI studies indicate that in the absence of reperfusion, at least a portion of mismatch tissue generally does proceed to infarction (Figure 2) ${ }^{13-15}$ Importantly, the fate of mismatch tissue can be altered by reperfusion, whether spontaneous or secondary to thrombolysis. ${ }^{16}$ A mismatch pattern may therefore be clinically useful as a marker for treatment potential.

Table 1: Perfusion and diffusion changes in penumbra and ischemic core tissue

\begin{tabular}{c|c|c}
\hline Perfusion Parameter & $\begin{array}{c}\text { Penumbra } \\
\text { (Potentially } \\
\text { Salvageable) }\end{array}$ & $\begin{array}{c}\text { Core } \\
\text { (Irreversibly } \\
\text { Injured) }\end{array}$ \\
\hline Cerebral Blood Flow (CBF) & $\downarrow$ & $\downarrow$ \\
\hline Cerebral Blood Volume (CBV) & $\uparrow / \leftrightarrow$ & $\downarrow$ \\
\hline Time to Peak (TTP) & $\uparrow$ & $\uparrow$ \\
\hline Mean Transit Time (MTT) & $\uparrow$ & $\uparrow$ \\
\hline $\begin{array}{c}\text { Tmax (TTP after } \\
\text { deconvolution) }\end{array}$ & $\uparrow$ & $\uparrow$ \\
\hline $\begin{array}{c}\text { Diffusion-Weighted Imaging } \\
\text { (MRI) }\end{array}$ & & \\
\hline $\begin{array}{c}\text { Diffusion-Weighted Image } \\
\text { Intensity }\end{array}$ & $\leftrightarrow$ & $\uparrow^{*}$ \\
\hline Apparent Diffusion Coefficient & $\leftrightarrow$ & $\downarrow *$ \\
\hline
\end{tabular}

* Areas with diffusion restriction (i.e. high signal on DWI and low ADC) may reverse if timely reperfusion occurs, making these areas penumbral in nature.

\section{Imaging the Ischemic Penumbra: CT Perfusion}

Estimates of the extent of infarction can also be made with non-contrast CT (NCCT). Irreversibly damaged tissue is visible on NCCT as areas of decreased attenuation, relative to normal parenchyma, due to the presence of cerebral edema. ${ }^{17}$ Unfortunately, hypo-attenuation in hyper-acute stroke is not universally present, making it less reliable than DWI for delineating infarct core. ${ }^{18}$ Adding computed tomography perfusion (CTP) to the imaging protocol for ischemic stroke permits visualization of both infarct core and hypoperfused tissue at risk. Cerebral tissue with very low CBV has been shown to be destined for infarction, due to failure of autoregulatory protective mechanisms. ${ }^{17,19-23}$ Regions where $\mathrm{CBV}$ is reduced are therefore irreversibly injured, consistent with ischemic core tissue (Table 1). Thus a mismatch between the volume of tissue with decreased blood flow (i.e. delayed Time to Peak (TTP) and/or low CBF) and that with low CBV can be used as a CTP-based operational definition of the ischemic penumbra (Figure 3). ${ }^{22-25}$ Similar to MRI, CTP-based mismatch definitions have also been shown to predict infarct growth in patients with persisting blood flow deficits. ${ }^{26,27}$

\section{The Mismatch Hypothesis: Current Evidence}

The hypothesis that selection of thrombolysis patients on the basis of MRI or CTP defined mismatch improves outcome in an extended therapeutic time window has not yet been definitively proven. Nonetheless, many clinical centres around the world have begun to utilize PWI-DWI, or CTP, in the selection of potential acute stroke thrombolysis patients. ${ }^{28}$ There is substantial evidence that penumbral selection is feasible and that it 'enriches' the treatment response, particularly when thrombolysis is administered more than three hours after onset, a time window normally associated with a lower success rate. ${ }^{29}$

A number of open label studies have demonstrated that it is feasible to select patients for thrombolytic therapy on the basis of MRI characteristics..$^{28,30,31}$ In one study, patients with a minimum of $20 \%$ PWI-DWI mismatch treated with tissue plasminogen activator (tPA) within six hours of onset experienced significant attenuation of infarct expansion, relative to historical controls. ${ }^{31}$ Although this was not a randomized study, it certainly supported the hypothesis that mismatch tissue represents penumbra and that the natural history can indeed be altered by thrombolysis. Another comparison of 1210 patients selected for tPA treatment within three hours of onset, on the basis of either NCCT $(n=714)$ or PWI-DWI mismatch $(n=316$; minimum of $20 \%$ assessed with TTP maps), indicated lower rates of hemorrhagic transformation were associated with the use of penumbral selection. ${ }^{28}$

The diffusion and perfusion imaging evaluation for understanding stroke evolution (DEFUSE) study was an open label investigation of the effects of tPA treatment in ischemic stroke patients three to six hours after symptom onset. ${ }^{32}$ All patients were imaged with PWI and DWI prior to treatment, but enrolment was not based on the results of either of these studies. The study tested the hypothesis that the clinical response associated with reperfusion following tPA, would be observed more frequently in patients with a minimum of $20 \%$ PWI-DWI mismatch. Despite the relatively small sample size $(n=74)$, patients with mismatch were five times more likely to recover 


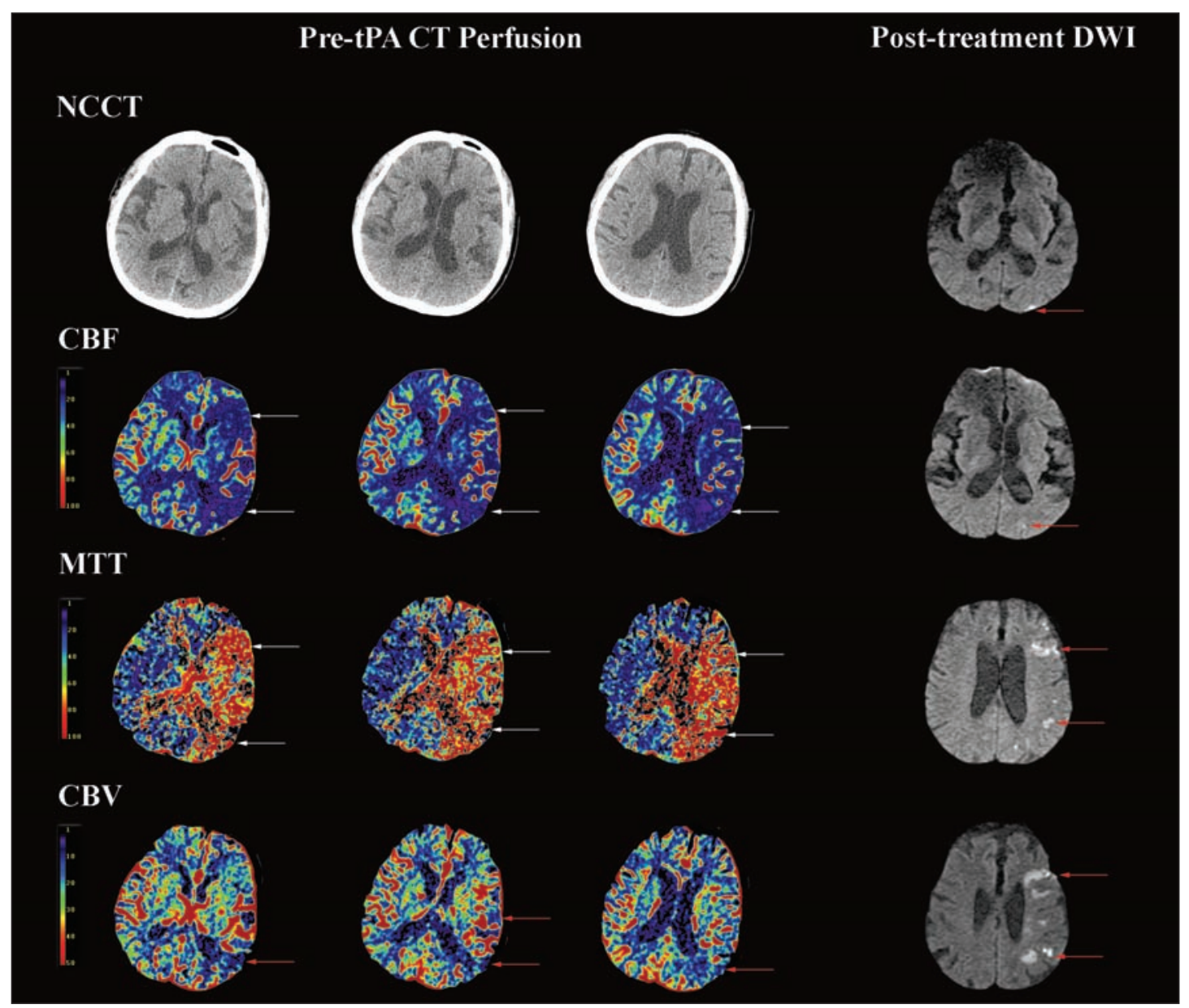

Figure 3: CT Perfusion (CTP) mismatch pattern in an acute ischemic stroke prior to treatment with tissue plasminogen activator (tPA) 5.5 h after symptom onset. The non-contrast CT (NCCT) reveals no ischemic changes. Cerebral Blood Flow (CBF; ml/100 g/min) is reduced and Mean Transit Time (MTT, 1/10th s) is prolonged in the left hemisphere (white arrows). Cerebral Blood Volume (CBV) is decreased posteriorly only (red arrows). This patient therefore had MTT/CBF-CBV and MTT/CBF-NCCT mismatch. Post-treatment diffusion-weighted MRI (DWI) demonstrates diffuse infarction in the hypoperfused tissue region. Post-tPA perfusion-weighted MRI (not shown) indicated complete reperfusion.

(modified Rankin Score $<2$, i.e. independent) than those without mismatch. ${ }^{32}$ In addition, the DEFUSE study demonstrated that patients with very large DWI lesions $(>100 \mathrm{ml})$ did not benefit from tPA, irrespective of the presence of mismatch. Furthermore, these patients were at increased risk of hemorrhagic transformation following treatment.

The echoplanar imaging thrombolysis evaluation trial (EPITHET) was a placebo controlled double-blind randomized study, designed specifically to test the mismatch hypothesis. ${ }^{33}$ One-hundred patients were randomized to placebo/tPA following DWI and PWI. Patients were not selected on the basis of MRI criteria, but were classified as mismatch/non-mismatch following randomization. The primary hypothesis was that tPA treatment would attenuate infarct expansion, assessed as the difference between acute DWI and day 90 T2-weighted image lesion volume, in patients with mismatch (Tmax+2s:DWI ratio $>1.2$ ). Although the primary endpoint was not significantly different between tPA and placebo treated patients, infarct growth was $30 \%$ smaller in the treatment group. ${ }^{33}$ In addition tPA treatment was associated with a significant $23 \%$ reduction in the proportion of mismatch patients who experienced infarct growth. The primary EPITHET endpoint was dependent on the ability of tPA to effectively restore blood flow. In mismatch patients with reperfusion, infarct growth was significantly attenuated. This demonstrates once again that the natural history of mismatch tissue is to proceed to infarction and that this can be reversed with effective reperfusion therapy. While some in the academic stroke community have dismissed the entire penumbral selection concept on the basis of a non-significant p-value in a small trial, the biological soundness of this approach to management is in fact supported by the EPITHET results and further studies are definitely warranted.

A series of studies assessing the novel thrombolytic desmoteplase, in a three to nine hour post onset treatment 
window, included either PWI-DWI or CTP mismatch in the inclusion criteria. Investigators were asked to obtain perfusion imaging with either modality and visually assess mismatch, using any available perfusion maps that they chose. If the investigator felt that there was a mismatch of $20 \%$ or more, patients were enrolled to placebo or various doses of desmoteplase..$^{34-36}$ Although initial studies appeared promising, the phase III study did not demonstrate a significant difference between treatment and placebo groups. ${ }^{36}$ The results of this study should not be viewed as a lack of evidence for the mismatch hypothesis. A post-hoc analysis indicated that many patients with very mild strokes were included, disproportionately in the placebo group. It is apparent that the definition of mismatch utilized in this trial was insufficient to identify patients at risk of infarct growth. In addition, it must be pointed out that the primary aim of this trial was not to test the mismatch hypothesis, but to demonstrate the efficacy of an unproven thrombolytic agent.

In many centres, CTP mismatch assessment has found it's way into routine imaging and thrombolysis protocols. ${ }^{37}$ There are retrospective studies demonstrating that mismatch identified with CTP is predictive of tissue salvage and clinical response to both IV and intra-arterial thrombolytic therapy. ${ }^{27,38}$ Mismatch criteria were utilized in the final desmoteplase trial described above and one other prospective assessment of tenectaplase in ischemic stroke patients presenting three to six hours after symptom onset. ${ }^{39} \mathrm{~A}$ trial with a design similar to EPITHET, is required to definitively test the hypothesis that CTP mismatch can be used to select optimal responders to thrombolysis.

\section{Obstacles to Penumbral Imaging in Acute Stroke Management}

The mismatch hypothesis is biologically plausible and although level Ia evidence for its utility in selecting potential thrombolysis patients remains elusive, it is being used in many centres. Nonetheless, utilization rates around the world are highly disparate. A number of logistical, technical and ideological factors have contributed to this situation.

\section{How do we define mismatch and how much is enough?}

At this point no accepted or standardized definition of what constitutes a significant mismatch pattern exists. As discussed in the accompanying article, multiple perfusion parameters, including TTP, CBF, Mean Transit Time (MTT), and Tmax (TTP after deconvolution), can be used to define tissue at risk. ${ }^{40,41} \mathrm{It}$ has not yet been established which of these parameters is best suited to defining acutely ischemic tissue. Optimal contrast bolus delay and dispersion correction algorithms, i.e. deconvolution, have yet to be determined.

It is also recognized that not all hypoperfused tissue will go on to infarct. There is a region of 'benign oligemia' surrounding the penumbra. ${ }^{12,16}$ Differentiating benign oligemia from penumbra is the key to the optimal mismatch definition, but this has proven to be exceedingly difficult in individual patients. ${ }^{41}$ The volume of putatively penumbral tissue will vary substantially with the threshold used to define significantly hypoperfused, i.e. non benign oligemic, tissue (Figures 4 and 5). ${ }^{41}$ As CBF (and MTT/TTP/Tmax) thresholds for infarction all
Table 2: MRI and CTP comparison

\begin{tabular}{|c|c|c|}
\hline & $\begin{array}{c}\text { MRI (PWI, DWI and } \\
\text { MRA) }\end{array}$ & CT (CTP and CTA) \\
\hline $\begin{array}{l}\text { Irreversibly } \\
\text { Injured Infarct } \\
\text { Core Imaging }\end{array}$ & $\begin{array}{l}\text { Very sensitive to early } \\
\text { ischemic changes }\end{array}$ & $\begin{array}{c}\text { CTA source images and } \\
\text { areas of low CBV are } \\
\text { correlated with DWI } \\
\text { changes }\end{array}$ \\
\hline Vessel Imaging & $\begin{array}{c}\text { Lower spatial resolution } \\
\text { than CTA } \\
\text { Intracranial MRA has } \\
\text { limited coverage, but } \\
\text { can be performed } \\
\text { without contrast }\end{array}$ & $\begin{array}{c}\text { Higher spatial resolution } \\
\text { than MRA and aortic arch } \\
\text { to cranial vertex vessels } \\
\text { can be imaged with one } \\
\text { exam }\end{array}$ \\
\hline $\begin{array}{l}\text { Blood Flow } \\
\text { Imaging }\end{array}$ & Whole brain coverage & $\begin{array}{l}\text { Limited to a } 4 \mathrm{~cm} \text { (in the } \\
\text { A-P direction) portion of } \\
\text { the brain on most scanners } \\
\text { currently in use }\end{array}$ \\
\hline Imaging Time & $\begin{array}{l}20 \text { minutes; additional } \\
\text { time required for } \\
\text { transfer of patient to } \\
\text { MRI suite and safety } \\
\text { screening } \\
\end{array}$ & $\begin{array}{l}\text { 5-10 minutes; can be } \\
\text { performed immediately } \\
\text { after NCCT }\end{array}$ \\
\hline Contrast Agent & $\begin{array}{l}\text { Gadolinium: Low risk } \\
\text { of allergic } \\
\text { reaction/toxicity; } \\
\text { nephrogenic systemic } \\
\text { fibrosis associated with } \\
\text { advanced renal failure }\end{array}$ & $\begin{array}{l}\text { Iodinated agents: Low } \\
\text { risk of toxicity, but higher } \\
\text { than MRI. Contrast } \\
\text { Induced Nephropathy risk } \\
\text { highest in patients with } \\
\text { pre-existing renal disease }\end{array}$ \\
\hline Radiation & None & $\begin{array}{l}\text { Effective radiation dose } \\
\text { varies with scanner; } \\
\text { additional exposure } \\
\text { associated with CTA/CTP } \\
\text { is reasonable in acute } \\
\text { stroke }\end{array}$ \\
\hline Contraindications & $\begin{array}{l}\text { Ferro-magnetic } \\
\text { implants/foreign bodies, } \\
\text { severe claustrophobia, } \\
\text { history of allergic } \\
\text { reaction/sensitivity to } \\
\text { gadolinium }\end{array}$ & $\begin{array}{l}\text { Severe renal failure, } \\
\text { history of allergic } \\
\text { reaction/sensitivity to } \\
\text { iodinated contrast agents }\end{array}$ \\
\hline
\end{tabular}

vary with duration of ischemia and tissue type, it is impossible to define the area at risk on the basis of any absolute flow value. ${ }^{16,42,43}$ Innumerable combinations of perfusion parameters and thresholds make a multitude of mismatch definitions possible in any given patient..$^{40,41}$ These are not merely theoretical considerations, as the choice of mismatch definition will result in the re-classification of many patients-which obviously has significant implications for both clinical trials and patient management.

What is very clear is that all definitions of mismatch to date have been far too liberal. The choice of a $20 \%$ threshold for defining mismatch is completely arbitrary and has no physiological correlates.$^{44,45}$ In fact, this is generally a very small amount of tissue and is of questionable clinical significance in most patients. The EPITHET study has also made it clear that even utilizing a deconvolved PWI parameter and a threshold 


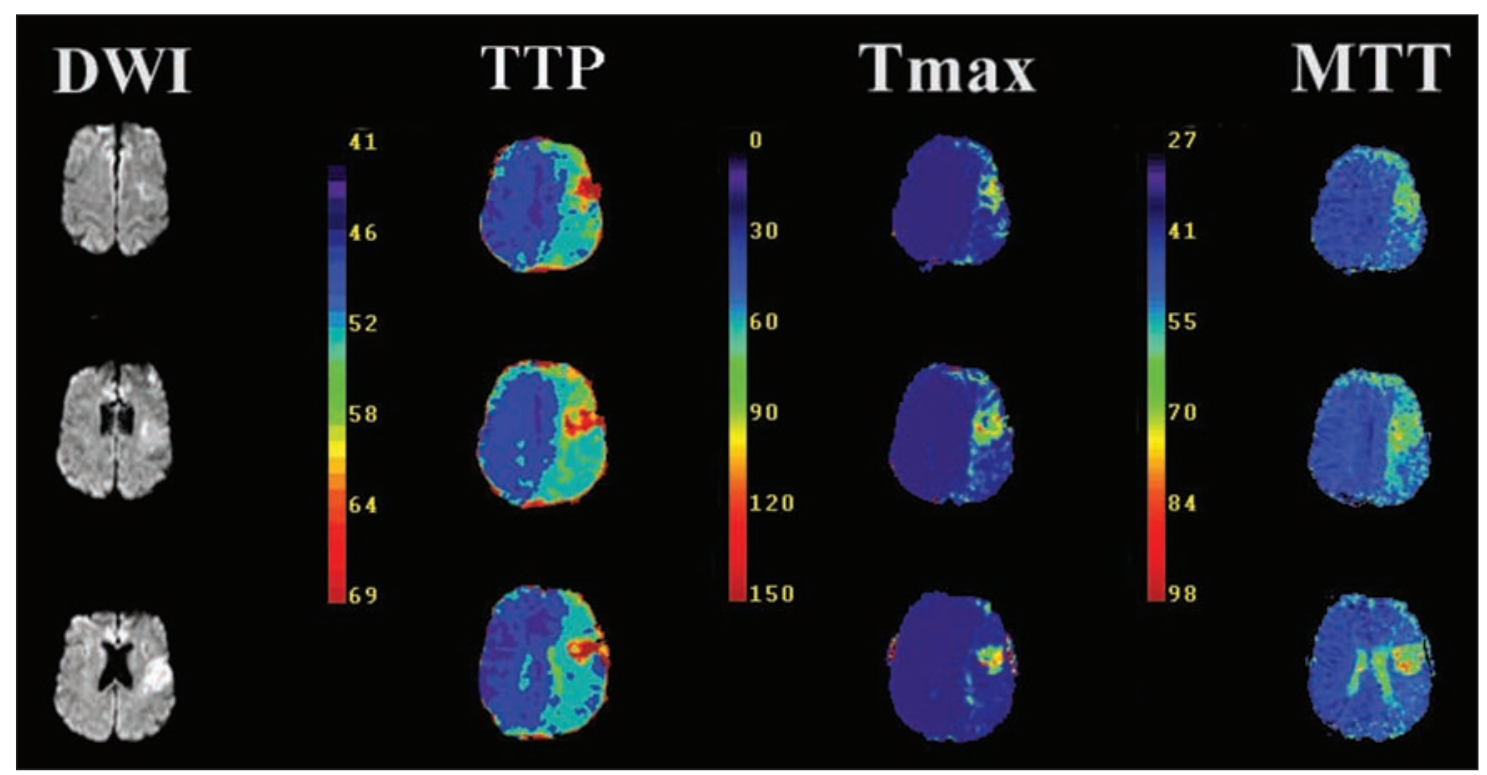

Figure 4: Image processing effects on the apparent volume of perfusion deficits in an acute stroke patient. Time to Peak (TTP) maps indicate hypoperfusion in the entire left hemisphere of this patient. Application of the deconvolution algorithm and calculation of Tmax or Mean Transit Time (MTT) maps, however, indicates that the true volume of hypoperfused tissue is much smaller. In addition, assessment of these maps in real time is limited to subjective assessment, as no threshold or planimetric volume measurements can be made on the console (compare to Figure 2). These factors do make objective mismatch assessment challenging in the acute setting.

$($ Tmax $+2 s)$, perfusion imaging frequently over-estimates the volume of at risk tissue, by including areas which are oligemic, but will never go on to infarct.

\section{Unrealistic expectations of mismatch models}

There are different solutions to the mismatch definition problems described above. The simplest is simply to increase the cut-off point for defining threshold. Post-hoc analyses of EPITHET and DEFUSE indicate that mismatch ratios of 200 to $260 \%$, rather than $20 \%$, identify the target patient population that will respond best to tPA. ${ }^{44,46}$ An alternative is to apply more stringent thresholds, i.e. Tmax $+8 \mathrm{~s}$, rather than $+2 \mathrm{~s}$, to the perfusion parameters, making it less likely that areas of benign oligemia will be identified as penumbral.

Both of these approaches lead to one unavoidable issue. Applying more conservative definitions of mismatch ultimately reduces the number of patients who are considered eligible for therapy. Having gone through the exercise of obtaining penumbral imaging, there is often a tendency to want to followup with treatment. This is very counter-intuitive, however, as there is very good evidence that both penumbral volumes and the response to treatment decrease exponentially with time. ${ }^{13,29}$ Thus, we should actually expect that a minority of patients imaged beyond 4.5 hours after symptom onset will have penumbral patterns. Nonetheless, in order to identify these patients it is necessary to assess CBF in all potential candidates. This is an even greater problem in clinical trials, where there is inexorable pressure to recruit patients as quickly as possible. Thus, there may be a tendency to 'over-call' mismatch or look for areas of penumbral tissue in patients who are unlikely to benefit. The two groups that seem to fall into this latter category are those with very small infarcts and patients with large parenchymal lesions, irrespective of the presence of mismatch (Figure 5). The former appears to have been a problem in particular for the latest desmoteplase trial. ${ }^{36}$ Finally, it is important to recognize that treatment does not guarantee complete salvage of mismatch tissue, even in the event of rapid reperfusion (Figure 4).

\section{Limited front-line perfusion and lesion volume assessment tools}

There is a significant discordance between the assessment of PWI and DWI in research studies and that practiced by physicians in the acute clinical setting. Studies utilize planimetric volume measurement tools and mathematical calculations to categorize patients as mismatch/non-mismatch. In contrast, clinicians are forced to make binary assessments (mismatch present or absent) based solely on the appearance of images on the console/PACS viewer. It has been demonstrated very clearly that clinicians can not reliably or even consistently assess the degree of mismatch with this type of approach to the images, and more objective definitions are required. ${ }^{47,48}$

The EPITHET and DEFUSE studies utilized an a priori definition of perfusion deficits; Tmax $+2 \mathrm{~s}$ threshold - that is only tissue with a delayed time to peak of contrast arrival after deconvolution of two seconds or more was considered to be at risk (Figure 5). While this PWI parameter provides a standardized and consistent measure of blood flow deficits, it is 


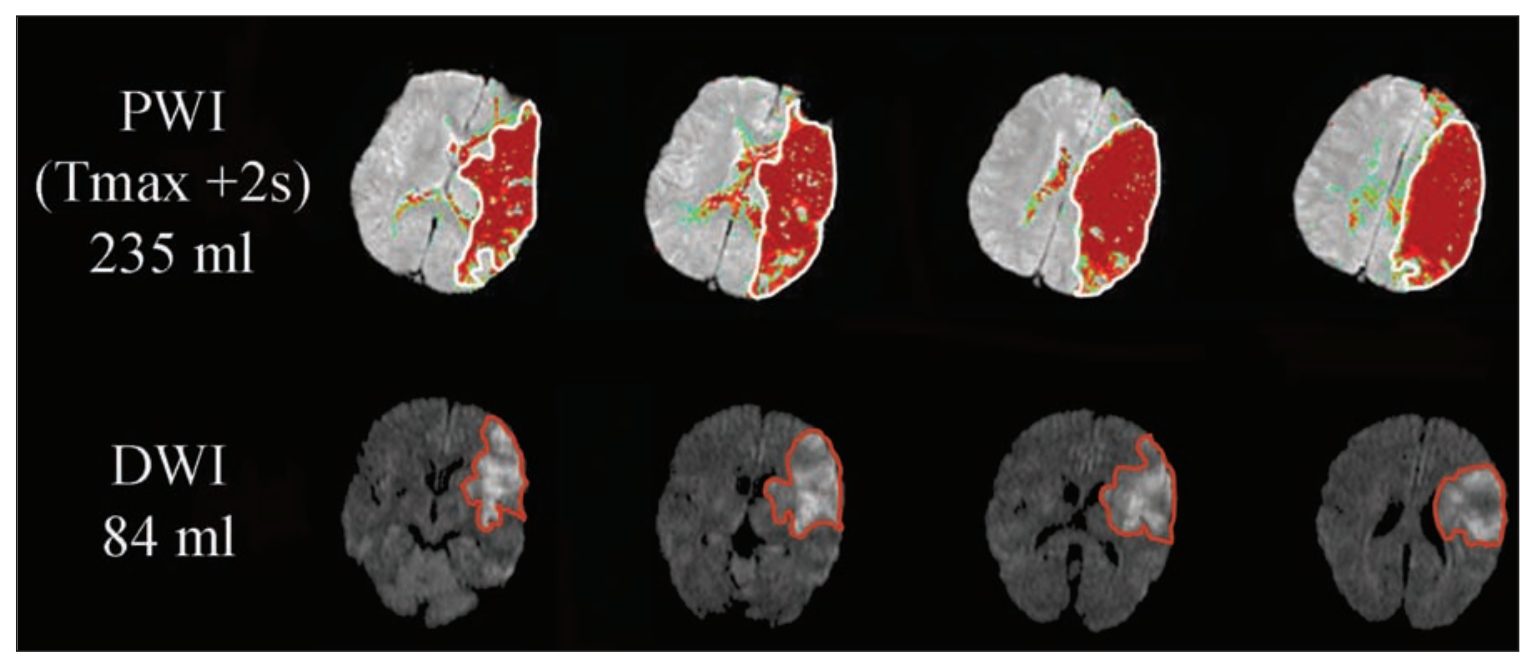

Figure 5: This patient does have objective PWI-DWI mismatch $($ Tmax $+2 \mathrm{~s}$ : DWI ratio $=2.8)$. Despite this, it is important to recognize that the large DWI lesion will result in significant clinical deficits, irrespective of successful treatment.

currently not available on software provided by MRI or CT manufacturers. None of the manufacturer-supplied software permits a threshold to be applied to PWI/CTP images. Thus, clinicians must rely on intensity/colour scales, which are often non-quantitative (relative), to visually estimate which areas are significantly hypoperfused (Figure 4). Software available on the console or workstation at many institutions does not even permit deconvolution, limiting assessment to TTP and relative CBF maps that will significantly over-estimate the volume of truly at risk tissue in many patients. ${ }^{41}$ In centres where deconvolution algorithms are available, this requires some operator input. Although this can be performed in a matter of minutes, it does require training and more importantly enthusiasm on the part of the clinicians, radiologists and technologists involved in the care of the patient.

An additional problem is the lack of accurate volume measurement tools available to the acute management team. Accurate stroke lesion volumes are obtained in imaging studies using planimetric techniques (Figure 5). Automated or semiautomated region of interest assessment tools may one day allow accurate stroke volume calculations in 'real time,' but this is generally not possible with currently available clinical CT or MRI software. A number of tools have been developed in order to assist clinicians and radiologists in rapidly assessing perfusion images in an objective and standardized fashion. One approach is to adapt the ASPECTS (Alberta Stroke Program Early CT Score $)^{49}$ system to PWI and DWI images. This has been demonstrated to accurately predict mismatch by volume. ${ }^{50}$ Similarly, ASPECT scores can be applied to CT perfusion images, thus providing a semi-quantitative estimate of penumbral tissue volume. ${ }^{23,51}$ A geometric approach, known as the $\mathrm{ABC} / 2$ method, is a validated technique for estimation of intracerebral hematoma volumes. ${ }^{52,53}$ Although this approach can also be applied to ischemic stroke lesions, reports are limited and the method has not yet been validated..$^{54}$

\section{Lack of engagement by clinicians}

It is clear that the mismatch hypothesis, while elegant has been over-simplified and practical implementation will require some investment of time and effort on the part of stroke clinicians. It is also evident that some familiarity with both imaging physics and cerebral blood flow regulation is required to interpret the results of penumbral investigations. This is currently not part of routine training for either clinicians or neuro-radiologists. In the absence of compelling evidence that these investigations will alter patient management, many clinicians have adopted an indifferent attitude towards blood flow imaging in particular. It is undoubtedly easier to treat patients on the basis of a pre-defined therapeutic time window, clinical findings and a NCCT. Obtaining and interpreting maps of blood flow often requires additional discussion between clinicians and radiologists. As outlined above, the technical challenges associated with these imaging studies also require time. While this additional time may not be justified in patients who are clearly eligible for tPA, it is absolutely essential if we are ever to offer therapy to patients in extended therapeutic windows, whether this be in an evidence-based fashion or empirically.

\section{MRI Versus CT Perfusion and CT Angiography}

There is considerable debate surrounding the optimal modality for assessing mismatch. ${ }^{55}$ It has been clearly demonstrated that MRI, specifically DWI, is more sensitive for detection of early ischemic changes. ${ }^{18,56}$ Using PWI and MRA, it is possible to obtain a comprehensive pathophysiological assessment, which may ultimately be used to guide thrombolytic and other tissue rescue therapies. In addition, concerns about the ability of MRI to detect intracranial hemorrhage are now known to be unwarranted. ${ }^{57,58}$ Therefore, in centres where rapid access protocols are in place, it is ideal to proceed directly to MRI in 
acute stroke. Nonetheless, MRI is generally less accessible hyper-acutely and always involves more imaging time. In the case of thrombolysis, where 'time is brain', these delays are critical and unacceptable. In addition, there will always be patients with contraindications to MRI, most notably those with pacemakers, ferro-magnetic implants and other foreign bodies.

The major advantage of CTP is the fact that it can be performed with NCCT as part of the initial stroke assessment, with minimal delay to therapy. Combined with CTA and NCCT, CTP provides a comprehensive multimodal stroke assessment, which accurately predicts tissue outcome..$^{22,23,25}$ Of course, this does require an established acute stroke assessment protocol, in order to avoid repeat trips to the scanner. Computed tomography perfusion does provide similar information to PWI, albeit in a more limited fashion. Unlike MRI, which is capable of imaging blood flow in the entire brain, CTP is limited to imaging within the extent of both the x-ray column and the detectors. Most multi-detector CT scanners currently in operation are limited by detector widths to CTP acquisition with only $2-4 \mathrm{~cm}$ cranialcaudal coverage of the brain. Thus, it is possible, that areas of hypoperfusion may not be recognized in acute stroke patients imaged with this technique, depending on placement of the CTP acquisition region. This can be overcome by performing two CTP exams in different locations, but this does result in further radiation and contrast exposure. ${ }^{59}$ This limitation has already been overcome by at least one CT manufacturer, however, as a multi-slice scanner capable of whole brain perfusion and simultaneous CTA, with a single contrast injection is now available..$^{60,61}$

Renal failure is relatively common in the acute stroke population, as hypertension and diabetes are risk factors shared by both conditions. Iodinated contrast agents do pose an increased risk of contrast induced nephropathy in these patients. However, a combined CTP-CTA protocol has been shown to be associated with very low (2\%) rates of contrast induced nephropathy, defined as a rise in serum creatinine of $>25 \%$, none of which led to chronic kidney disease. ${ }^{62}$ Contrast induced nephropathy rates were not higher in patients imaged without a known serum Creatinine. The Canadian Association of Radiologists guidelines indicate that in emergent situations, contrast media may be used prior to obtaining serum creatinine levels, provided there are no risk factors for renal impairment, such as pre-existing kidney disease or diabetes. ${ }^{63}$ In these cases it is recommended that patients be prophylactically hydrated with $300-500 \mathrm{ml}$ of IV crystalloid during transfer to the CT. There is also an association between severe renal impairment and a very rare reaction to gadolinium, known as nephrogenic systemic fibrosis. ${ }^{64}$ This is much less common than iodinated contrast induced nephropathy, but does need to be considered, particularly if the MRI contrast study is unlikely to change management.

What then is the imaging modality of choice in acute stroke? Clearly, either option is superior to NCCT alone. In the future, as imaging based algorithms for reperfusion strategies become more common, the utility of MRI versus advanced CT will likely vary with the available resources between different centres. Ultimately, access to both modalities will be required to maximize the number of patients in whom penumbral imaging can be performed, as a number of patients will have contraindications to either technique (Table 2).

\section{Alternative Penumbral Definitions}

Due to the relative lack of access to perfusion imaging modalities in many centres, attempts have been made to identify predictors of penumbral patterns, without actually imaging cerebral blood flow. These models all utilize a surrogate for tissue at risk, such as clinical deficits or an acute vessel occlusion, combined with evidence of limited parenchymal injury.

\section{Vessel occlusion models}

It has been hypothesized that vessel occlusion, identified with either CTA or MRA, co-localized with a lack of evidence of significant tissue injury will predict a penumbral pattern. Indeed, patients with large artery occlusion are more likely to have mismatch patterns. ${ }^{65}$ A post-hoc analysis of the DEFUSE study defined MRA-DWI mismatch as the presence of a visible occlusion on MRA and a DWI lesion volume $<25 \mathrm{ml}$. $^{66}$ This pattern was seen in 27 of 62 patients studied and reperfusion was associated with clinical improvement. An occluded artery in a patient with symptoms of acute stroke therefore appears to be a logical target for thrombolysis. Accordingly, vessel occlusion, identified on either CTA or MRA, combined with a lack of evidence of extensive early infarction, i.e. $>1 / 3$ of the middle cerebral artery territory, is being used as the primary inclusion criterion an ongoing randomized controlled trial of desmoteplase in acute stroke. ${ }^{67}$

The vessel occlusion model does minimize the need for image post-processing, as occlusions can be seen on source images. It also eliminates all of the difficulties associated with blood flow image interpretation, and is therefore appealing to many clinicians. Unfortunately, it has been demonstrated that many patients with significant penumbral patterns do not have visible occlusions on MRA. Computed tomogram angiography is likely to be superior in this regard, due to higher spatial resolution and coverage, but patients who still may benefit from thrombolysis will be excluded from therapy if selection is based on this model. Paradoxically, CTA and NCCT based therapy will likely include some patients with completed infarcts and no penumbra, due to the lower sensitivity of NCCT for hyperacute changes. Computed tomogram angiography source image inspection helps somewhat in this regard, as they have been shown to improve the sensitivity for detection of early ischemic changes, relative to NCCT alone. ${ }^{68-70}$ This is due to the fact that CTA source images are volume-weighted, making areas with low CBV more apparent. Nonetheless, this model has the potential to both under-treat and over-treat certain acute stroke populations and should not be viewed as a complete replacement for perfusion imaging.

\section{Clinical-Diffusion mismatch}

An alternative to the PWI-DWI mismatch definition of mismatch is to utilize clinical information in lieu of perfusion data. Clinical-diffusion mismatch (CDM) has been defined as the co-existence of a small $(\leq 25 \mathrm{ml})$ DWI lesion in the presence of significant neurological deficits (NIH Stroke Scale score $\geq 8){ }^{71}$ Accurate identification of CDM patterns requires planimetric measurement of the DWI volume, which makes real time use problematic. An alternative is to replace the DWI volume used in 
the current definition with a maximum DWI ASPECT score, i.e. $>7$. Clinical-diffusion mismatch based on DWI ASPECTS, has been shown to predict early neurological deterioration, consistent with the natural history of penumbral evolution. ${ }^{72}$ Direct comparisons with PWI-DWI mismatch have not been made, but it seems likely that this rapid assessment tool will also predict penumbral patterns with reasonable accuracy. Clinicaldiffusion mismatch has been shown to predict the presence of PWI-DWI mismatch with high specificity, suggesting it is a reasonable surrogate marker of the ischemic penumbra. ${ }^{73}$ However, similar to vessel occlusion models, many patients without CDM will have penumbral patterns and be excluded from therapy if perfusion imaging is not part of the assessment algorithm.

\section{A Practical Approach to Penumbral Imaging}

Despite all of the difficulties described above, imaging of the penumbra can be extremely useful to the treating physician. At the very least, definitive evidence of $\mathrm{CBF}$ abnormalities removes diagnostic uncertainty in cases of a normal CT and a clinical scenario consistent with a stroke mimic such as migraine, epilepsy or a conversion disorder. Although not universal, many clinicians will consider thrombolysis outside of established protocols, the most notable exception being patients presenting outside the therapeutic window of 4.5 hours after onset. This is known to be associated with higher risk of hemorrhagic complications. ${ }^{74}$ It therefore behoves the clinician to ensure there is a likelihood of benefit. If penumbral imaging is used in this decision making process, it is important to recognize which perfusion $\operatorname{map}(\mathrm{s})$ are being assessed and what each represents (Table 1). It should always be remembered that these maps have a tendency to over-estimate the true volume of tissue at risk. Thrombolysis should be restricted to patients with very large mismatch patterns (Figures 1 and 2). Patients with limited blood flow deficits or large established infarct core areas seen on DWI or CBV maps should not be treated (Figure 5).

\section{Conclusions}

It appears likely that in the future penumbral imaging will become increasingly important in selecting stroke patients for therapy beyond the current therapeutic time window. The most promising group of patients are those in which the temporal course of events is unknown, such as patients who are found dysphasic or who wake with symptoms. There are other compelling reasons to image blood flow in all acute stroke patients much more routinely. Changes in $\mathrm{CBF}$ are the fundamental basis of the stroke pathophysiology. Cerebral blood flow assessment makes it possible to make rational management decisions that are based on patient specific pathology, rather than broad clinical criteria that may not apply to individual cases. Although this will require a significant investment on the part of all involved in the care of these patients, it is a sound one that will undoubtedly result in improved patient care.

Ultimately, simplified and automated CBF assessment tools will be required to facilitate widespread utilization of these imaging modalities. These are being developed presently, although the concept of a 'treat/do not treat' map is still theoretical at the moment. Even if this does become possible in the future, clinicians would be well advised to understand the concepts underlying penumbral assessment, rather than treating it as a 'black box'. In the meantime, the clinical stroke community must lead the way in the development and utilization of CBF imaging. Cases of diagnostic uncertainty should be viewed as opportunities to become familiar with the MRI and/or CTP/CTA tools presently available.

\section{ACKNOWLEDGEMENTS}

Dr. Butcher is supported by New Investigator Awards and project grant funding from the Alberta Heritage Foundation for Medical Research, Canadian Institutes for Health Research and Heart and Stroke Foundations of Canada and Alberta, NWT, and Nunavut.

\section{REFERENCES}

1. Astrup J, Symon L, Branston NM, Lassen NA. Cortical evoked potential and extracellular $\mathrm{K}+$ and $\mathrm{H}+$ at critical levels of brain ischemia. Stroke. 1977 Jan-Feb;8(1):51-7.

2. Symon L, Branston NM, Strong AJ. The concept of thresholds of ischemia in relation to brain structure and function. J Clin Pathol. 1977;30(S11):149-54.

3. Astrup J, Siesjo BK, Symon L. Thresholds in cerebral ischemia the ischemic penumbra. Stroke. 1981 Nov-Dec;12(6):723-5.

4. Marchal G, Beaudouin V, Rioux P, de la Sayette V, Le Doze F, Viader F, et al. Prolonged persistence of substantial volumes of potentially viable brain tissue after stroke: a correlative PET-CT study with voxel-based data analysis. Stroke. 1996 Apr;27(4): 599-606.

5. Marchal G, Furlan M, Beaudouin V, Rioux P, Hauttement JL, Serrati C, et al. Early spontaneous hyperperfusion after stroke. A marker of favourable tissue outcome? Brain. 1996 Apr;119 (Pt 2):409-19.

6. Heiss WD. Ischemic penumbra: evidence from functional imaging in man. J Cereb Blood Flow Metab. 2000 Sep;20(9):1276-93.

7. Donnan GA, Dewey HM, Davis SM. MRI and stroke: why has it taken so long? Lancet. 2007 Jan 27;369(9558):252-4.

8. Schellinger PD, Fiebach JB. Perfusion-weighted imaging/diffusionweighted imaging mismatch on MRI can now be used to select patients for recombinant tissue plasminogen activator beyond 3 hours: pro. Stroke. 2005 May;36(5):1104-5.

9. Parsons MW, Pepper EM, Bateman GA, Wang Y, Levi CR. Identification of penumbra and infarct core on hyperacute noncontrast and perfusion CT. Neurology. 2007 March;68(3):730-6.

10. Davis SM, Donnan GA, Butcher KS, Parsons M. Selection of thrombolytic therapy beyond $3 \mathrm{~h}$ using magnetic resonance imaging. Curr Opin Neurol. 2005 Feb;18(1):47-52.

11. Schlaug G, Benfield A, Baird AE, Siewert B, Lovblad KO, Parker $\mathrm{RA}$, et al. The ischemic penumbra: operationally defined by diffusion and perfusion MRI. Neurology. 1999;53(7):1528-37.

12. Kidwell CS, Alger JR, Saver JL. Beyond mismatch: evolving paradigms in imaging the ischemic penumbra with multimodal magnetic resonance imaging. Stroke. 2003;34 (11):2729-35.

13. Darby DG, Barber PA, Gerraty RP, Desmond PM, Yang Q, Parsons $\mathrm{M}$, et al. Pathophysiological topography of acute ischemia by combined diffusion-weighted and perfusion MRI. Stroke. 1999; 30(10):2043-52.

14. Barber PA, Darby DG, Desmond PM, Yang Q, Gerraty RP, Jolley $\mathrm{D}$, et al. Prediction of stroke outcome with echoplanar perfusionand diffusion-weighted MRI. Neurology. 1998 Aug;51(2): 418-26.

15. Warach S, Dashe JF, Edelman RR. Clinical outcome in ischemic stroke predicted by early diffusion-weighted and perfusion magnetic resonance imaging: a preliminary analysis. J Cereb Blood Flow Metab. 1996;16(1):53-9.

16. Butcher K, Parsons M, Baird T, Barber A, Donnan G, Desmond P, et al. Perfusion thresholds in acute stroke thrombolysis. Stroke. 2003;34:2159-64. 
17. Butcher KS, Lee SB, Parsons MW, Allport L, Fink J, Tress B, et al. Differential prognosis of isolated cortical swelling and hypoattenuation on CT in acute stroke. Stroke. 2007 Mar;38(3): 941-7.

18. Barber PA, Darby DG, Desmond PM, Gerraty RP, Yang Q, Li T, et al. Identification of major ischemic change. Diffusion-weighted imaging versus computed tomography. Stroke. 1999;30(10): 2059-65.

19. Eastwood JD, Lev MH, Azhari T, Lee TY, Barboriak DP, Delong DM, et al. CT perfusion scanning with deconvolution analysis: pilot study in patients with acute middle cerebral artery stroke. Radiology. 2002 Jan;222(1):227-36.

20. Smith WS, Roberts HC, Chuang NA, Ong KC, Lee TJ, Johnston SC, et al. Safety and feasibility of a CT protocol for acute stroke: combined CT, CT angiography, and CT perfusion imaging in 53 consecutive patients. AJNR Am J Neuroradiol. 2003 Apr;24(4): 688-90.

21. Kudo K, Terae S, Katoh C, Oka M, Shiga T, Tamaki N, et al. Quantitative cerebral blood flow measurement with dynamic perfusion CT using the vascular-pixel elimination method: comparison with $\mathrm{H}_{2}(15) \mathrm{O}$ positron emission tomography. AJNR Am J Neuroradiol. 2003 Mar;24(3):419-26.

22. Murphy BD, Fox AJ, Lee DH, Sahlas DJ, Black SE, Hogan MJ, et al. Identification of penumbra and infarct in acute ischemic stroke using computed tomography perfusion-derived blood flow and blood volume measurements. Stroke. 2006 Jul;37(7): 1771-7.

23. Parsons MW, Pepper EM, Chan V, Siddique S, Rajaratnam S, Bateman GA, et al. Perfusion computed tomography: prediction of final infarct extent and stroke outcome. Ann Neurol. 2005 Nov;58(5):672-9.

24. Wintermark M, Reichhart M, Cuisenaire O, Maeder P, Thiran JP, Schnyder P, et al. Comparison of admission perfusion computed tomography and qualitative diffusion- and perfusion-weighted magnetic resonance imaging in acute stroke patients. Stroke. 2002 Aug;33(8):2025-31.

25. Wintermark M, Reichhart M, Thiran JP, Maeder P, Chalaron M, Schnyder P, et al. Prognostic accuracy of cerebral blood flow measurement by perfusion computed tomography, at the time of emergency room admission, in acute stroke patients. Ann Neurol. 2002 Apr;51(4):417-32.

26. Silvennoinen HM, Hamberg LM, Lindsberg PJ, Valanne L, Hunter GJ.CT perfusion identifies increased salvage of tissue in patients receiving intravenous recombinant tissue plasminogen activator within 3 hours of stroke onset. AJNR Am J Neuroradiol. 2008 Jun;29(6):1118-23.

27. Parsons MW, Pepper EM, Bateman GA, Wang Y, Levi CR. Identification of the penumbra and infarct core on hyperacute noncontrast and perfusion CT. Neurology. 2007 Mar 6;68(10): 730-6.

28. Schellinger PD, Thomalla G, Fiehler J, Kohrmann M, Molina CA, Neumann-Haefelin T, et al. MRI-based and CT-based thrombolytic therapy in acute stroke within and beyond established time windows: an analysis of 1210 patients. Stroke. 2007 Oct;38(10):2640-5.

29. Hacke W, Donnan G, Fieschi C, Kaste M, von Kummer R, Broderick JP, et al. Association of outcome with early stroke treatment: pooled analysis of ATLANTIS, ECASS, and NINDS rt-PA stroke trials. Lancet. 2004 Mar 6;363(9411):768-74.

30. Schellinger PD, Jansen O, Fiebach JB, Heiland S, Steiner T, Schwab S, et al. Monitoring intravenous recombinant tissue plasminogen activator thrombolysis for acute ischemic stroke with diffusion and perfusion MRI. Stroke. 2000;31(6):1318-28.

31. Parsons MW, Barber PA, Chalk J, Darby DG, Rose S, Desmond PM, et al. Diffusion- and perfusion-weighted MRI response to thrombolysis in stroke. Ann Neurol. 2002 Jan;51(1):28-37.

32. Albers GW, Thijs VN, Wechsler L, Kemp S, Schlaug G, Skalabrin $\mathrm{E}$, et al. Magnetic resonance imaging profiles predict clinical response to early reperfusion: the diffusion and perfusion imaging evaluation for understanding stroke evolution (DEFUSE) study. Ann Neurol. 2006 Nov;60(5):508-17.
33. Davis SM, Donnan GA, Parsons MW, Levi C, Butcher KS, Peeters $\mathrm{A}$, et al. Effects of alteplase beyond $3 \mathrm{~h}$ after stroke in the Echoplanar Imaging Thrombolytic Evaluation Trial (EPITHET): a placebo-controlled randomised trial. Lancet Neurol. 2008 Apr; 7(4):299-309.

34. Furlan AJ, Eyding D, Albers GW, Al-Rawi Y, Lees KR, Rowley $\mathrm{HA}$, et al. Dose Escalation of Desmoteplase for Acute Ischemic Stroke (DEDAS): evidence of safety and efficacy 3 to 9 hours after stroke onset. Stroke. 2006 May;37(5):1227-31.

35. Hacke W, Albers G, Al-Rawi Y, Bogousslavsky J, Davalos A, Eliasziw M, et al. The Desmoteplase in Acute Ischemic Stroke Trial (DIAS): a phase II MRI-based 9-hour window acute stroke thrombolysis trial with intravenous desmoteplase. Stroke. 2005 Jan;36(1):66-73.

36. Hacke W, Furlan AJ, Al-Rawi Y, Davalos A, Fiebach JB, Gruber F, et al. Intravenous desmoteplase in patients with acute ischaemic stroke selected by MRI perfusion-diffusion weighted imaging or perfusion CT (DIAS-2): a prospective, randomised, doubleblind, placebo-controlled study. Lancet Neurol. 2009 Feb;8(2): $141-50$

37. Parsons MW. Perfusion CT: is it clinically useful? Int J Stroke. 2008 Feb;3(1):41-50.

38. Gasparotti R, Grassi M, Mardighian D, Frigerio M, Pavia M, Liserre R, et al. Perfusion CT in patients with acute ischemic stroke treated with intra-arterial thrombolysis: predictive value of infarct core size on clinical outcome. AJNR Am J Neuroradiol. 2009 Apr; 30(4):722-7.

39. Parsons MW, Miteff F, Bateman GA, Spratt N, Loiselle A, Attia J, et al. Acute ischemic stroke: imaging-guided tenecteplase treatment in an extended time window. Neurology. 2009 Mar 10; 72(10):915-21.

40. Hjort N, Butcher K, Davis S, Kidwell CS, Koroshetz WJ, Rother J, et al. Magnetic resonance imaging criteria for thrombolysis in acute cerebral infarct. Stroke. 2005;36(2):388-97.

41. Butcher KS, Parsons M, MacGregor L, Barber PA, Chalk J, Bladin $\mathrm{C}$, et al. Refining the perfusion-diffusion mismatch hypothesis. Stroke. 2005 May 12, 2005:1153-9.

42. Hossman K. Viability thresholds and the penumbra of focal ischemia. Ann Neurol. 1994;36:557-65.

43. Arakawa S, Wright PM, Koga M, Phan TG, Reutens DC, Lim I, et al. Ischemic thresholds for gray and white matter: a diffusion and perfusion magnetic resonance study. Stroke. 2006 May;37(5): 1211-6.

44. Kakuda W, Lansberg MG, Thijs VN, Kemp SM, Bammer R, Wechsler LR, et al. Optimal definition for PWI/DWI mismatch in acute ischemic stroke patients. J Cereb Blood Flow Metab. 2008 May;28(5):887-91.

45. Donnan GA, Baron JC, Ma H, Davis SM. Penumbral selection of patients for trials of acute stroke therapy. Lancet Neurol. 2009 Mar;8(3):261-9.

46. Christensen S, Parsons M, DeSilva D, Ebinger M, Butcher K, Fink $\mathrm{J}$, et al. Optimal mismatch definitions for detecting treatment response in acute stroke. Cerebrovascular Dis. 2008;25 Suppl 2:33.

47. Coutts SB, Simon JE, Tomanek AI, Barber PA, Chan J, Hudon ME, et al. Reliability of assessing percentage of diffusion-perfusion mismatch. Stroke. 2003 Jul;34(7):1681-3.

48. Butcher KS, Parsons MW, Davis S, Donnan G. PWI/DWI mismatch: better definition required. Stroke. 2003 Nov;34(11): e215-6; author reply e-6.

49. Pexman JHW, Barber PA, Hill MD, Sevick RJ, Demchuk AM, Hudon ME, et al. Use of the Alberta Stroke Program Early CT Score (ASPECTS) for assessing CT Scans in patients with acute stroke. AJNR Am J Neuroradiol. 2001; 22(8):1534-42.

50. Butcher K, Parsons M, Allport L, Lee SB, Barber PA, Tress B, et al. Rapid assessment of perfusion-diffusion mismatch. Stroke. 2008 Jan;39(1):75-81.

51. Aviv RI, Mandelcorn J, Chakraborty S, Gladstone D, Malham S, Tomlinson G, et al. Alberta Stroke Program Early CT Scoring of CT perfusion in early stroke visualization and assessment. AJNR Am J Neuroradiol. 2007 Nov-Dec;28(10):1975-80. 
52. Kothari RU, Brott T, Broderick JP, Barsan WG, Sauerbeck LR, Zuccarello $\mathrm{M}$, et al. The ABCs of measuring intracerebral hemorrhage volumes. Stroke. 1996;27:1304-5.

53. Kwak R, Kadoya S, Suzuki T. Factors affecting the prognosis in thalamic hemorrhage. Stroke. 1983 Jul-Aug;14(4):493-500.

54. Gomez-Marino R, Andre C, Novis SA. [Volumetric determination of cerebral infarction in the acute phase using skull computed tomography without contrast: comparative study of 3 methods]. Arq Neuropsiquiatr. 2001 Jun;59(2-B):380-3.

55. Lev MH. CT versus MR for acute stroke imaging: is the "obvious" choice necessarily the correct one? AJNR Am J Neuroradiol. 2003 Nov-Dec;24(10):1930-1.

56. Lansberg MG, Albers GW, Beaulieu C, Marks MP. Comparison of diffusion-weighted MRI and CT in acute stroke. Neurology. 2000 Apr 25;54(8):1557-61.

57. Schellinger PD, Jansen O, Fiebach JB, Hacke W, Sartor K. A standardized MRI stroke protocol: comparison with CT in hyperacute intracerebral hemorrhage. Stroke. 1999;30(4):765-8.

58. Kidwell CS, Chalela JA, Saver JL, Starkman S, Hill MD, Demchuk AM, et al. Comparison of MRI and CT for detection of acute intracerebral hemorrhage. JAMA. 2004 Oct;292(15):1823-30

59. Mnyusiwalla A, Aviv RI, Symons SP. Radiation dose from multidetector row CT imaging for acute stroke. Neuroradiology. Epub 2009 Jun 9.

60. Klingebiel R, Siebert E, Diekmann S, Wiener E, Masuhr F, Wagner $\mathrm{M}$, et al. 4-D Imaging in cerebrovascular disorders by using 320slice CT: feasibility and preliminary clinical experience. Acad Radiol. 2009 Feb;16(2):123-9.

61. Siebert E, Bohner G, Dewey M, Masuhr F, Hoffmann KT, Mews J, et al. 320-slice CT neuroimaging: initial clinical experience and image quality evaluation. Br J Radiol. 2009 Jul;82(979):561-70.

62. Hopyan JJ, Gladstone DJ, Mallia G, Schiff J, Fox AJ, Symons SP, et al. Renal safety of CT angiography and perfusion imaging in the emergency evaluation of acute stroke. AJNR Am J Neuroradiol. 2008 Nov;29(10):1826-30.

63. Benko A, Fraser-Hill M, Magner P, Capusten B, Barrett B, Myers A, et al. Canadian Association of Radiologists: consensus guidelines for the prevention of contrast-induced nephropathy. Can Assoc Radiol J. 2007 Apr;58(2):79-87.

64. Pedersen M. Safety update on the possible causal relationship between gadolinium-containing MRI agents and nephrogenic systemic fibrosis. J Magn Reson Imaging. 2007 May;25(5): 881-3.
65. Barber PA, Davis SM, Darby DG, Desmond PM, Gerraty RP, Yang $\mathrm{Q}$, et al. Absent middle cerebral artery flow predicts the presence and evolution of the ischemic penumbra. Neurology. 1999 Apr $12 ; 52(6): 1125-32$

66. Lansberg MG, Thijs VN, Bammer R, Olivot JM, Marks MP, Wechsler LR, et al. The MRA-DWI mismatch identifies patients with stroke who are likely to benefit from reperfusion. Stroke. 2008 Sep;39(9):2491-6.

67. Hill MD. Desmoteplase and imaging science. Lancet Neurol. 2009 Feb;8(2):126-8.

68. Ezzeddine MA, Lev MH, McDonald CT, Rordorf G, Oliveira-Filho J, Aksoy FG, et al. CT angiography with whole brain perfused blood volume imaging: added clinical value in the assessment of acute stroke. Stroke. 2002 Apr;33(4):959-66.

69. Lev MH, Segal AZ, Farkas J, Hossain ST, Putman C, Hunter GJ, et al. Utility of perfusion-weighted CT imaging in acute middle cerebral artery stroke treated with intra-arterial thrombolysis: prediction of final infarct volume and clinical outcome. Stroke. $2001 \mathrm{Sep} ; 32(9): 2021-8$.

70. Hunter GJ, Hamberg LM, Ponzo JA, Huang-Hellinger FR, Morris PP, Rabinov J, et al. Assessment of cerebral perfusion and arterial anatomy in hyperacute stroke with three-dimensional functional CT: early clinical results. AJNR Am J Neuroradiol. 1998;19:29-37.

71. Davalos A, Blanco M, Pedraza S, Leira R, Castellanos M, Pumar $\mathrm{JM}$, et al. The clinical-DWI mismatch: a new diagnostic approach to the brain tissue at risk of infarction. Neurology. 2004 Jun 22;62(12):2187-92

72. Tei H, Uchiyama S, Usui T. Clinical-diffusion mismatch defined by NIHSS and ASPECTS in non-lacunar anterior circulation infarction. J Neurol. 2007 Mar;254(3):340-6.

73. Prosser J, Butcher K, Allport L, Parsons M, MacGregor L, Desmond $\mathrm{P}$, et al. Clinical-diffusion mismatch predicts the putative penumbra with high specificity. Stroke. 2005 Aug;36 (8):1700-4.

74. Buchan AM, Barber PA, Newcommon N, Karbalai HG, Demchuk AM, Hoyte KM, et al. Effectiveness of t-PA in acute ischemic stroke: outcome relates to appropriateness. Neurology. $2000 \mathrm{Feb}$ 8;54(3):679-84. 